\title{
Erythematous candidiasis leading to systemic manifestations of human immunodeficiency virus co-infection with secondary syphilis: A diagnostic and therapeutic dilemma
}

\author{
Swastika Suvirya ${ }^{1}$, Rahul Gandhi ${ }^{2}$, Jyotsana Agarwal $^{3}$, Ranjitkumar Patil ${ }^{2}$
}

Correspondence: Dr. Rahul Gandhi

Email: drrahulgandhi@yahoo.com

\author{
'Department of Dermatology, Venereology and Leprosy, \\ King Georges Medical University, Lucknow, \\ Uttar Pradesh, India, \\ 2Department of Oral Medicine and Radiology, King \\ Georges Medical University, Lucknow, Uttar Pradesh, \\ India, \\ ${ }^{3}$ Department of Microbiology, King Georges Medical \\ University, Lucknow, Uttar Pradesh, India
}

\section{ABSTRACT}

The intensification of human immunodeficiency virus (HIV) and rising frequency of immunocompromised individuals have resulted in a resurgence of opportunistic infections. The most common opportunistic oral fungal infection in HIV-positive individuals is oral candidiasis. The classical presentation is as white scrapable form called as thrush, which is easily diagnosed and treated. The clinician is presented with a diagnostic and management dilemma when these lesions appear in new facades such as erythematous candidiasis, the latter's prevalence with HIV and AIDS being well established. In this case report, we present a case of Erythematous Candidiasis, which was associated with type $1 \mathrm{HIV}$ co-infected with syphilis. We highlight the diagnostic importance of a naive looking manifestation of the tongue which was followed by a series of challenging presentations of secondary syphilis. Since the patient had a negative Veneral Disease Research Laboratory and left us with a management dilemma, the article also features the importance of prozone phenomenon (seen in $2 \%$ cases of secondary syphilis), and it's higher association with HIV co-infected individuals. With confusing clinical oral manifestations associated with these diseases, the dentist might be the first person to encounter such lesions, who should be able to recognize erythematous candidiasis and correlate them with the underlying pathology.

Key words: Erythematous candidiasis, human immunodeficiency virus, prozone phenomenon, secondary syphilis, Treponema pallidum particle agglutination assay, venereal disease research laboratory

\section{INTRODUCTION}

The oral cavity can be rightly called as a "mirror", reflecting health and diseased states. It acts as a doorway to the body, serving as an embankment from the constant bombardment of bacteria, virus, and fungi from the external environment. In diseased states, the oral presentations are sometimes the first and maybe the only manifestation of underlying disease, especially in immunocompromised conditions. ${ }^{[1]}$ Often described as being "a disease of the diseased," Oral Candidiasis is markedly associated with immunocompromised states as human immunodeficiency virus (HIV), some studies reporting $90 \%$ occurrence of oral candidiasis in HIV infected individuals. ${ }^{[2]}$ Oral candidiasis is one of the seven cardinal lesions indicating towards a strong association of the former with HIV. Hence, the oral diagnostician must treat these lesions with suspicion and tread carefully in a direction pointing toward an

This is an open access article distributed under the terms of the Creative Commons Attribution-NonCommercial-ShareAlike 3.0 License, which allows others to remix, tweak, and build upon the work non-commercially, as long as the author is credited and the new creations are licensed under the identical terms.

For reprints contact: reprints@medknow.com

How to cite this article: Suvirya S, Gandhi R, Agarwal J, Patil R. Erythematous candidiasis leading to systemic manifestations of human immunodeficiency virus co-infection with secondary syphilis: A diagnostic and therapeutic dilemma. Eur J Dent 2015;9:449-52.

DOI: $10.4103 / 1305-7456.163219$ 
underlying condition. The immune system plays an important role in protecting individuals and when it is downcast, along with an altered social history, can lead to infections with those microbiologic agents in which the primary mode of transmission is sexual contact. Syphilis is a chronic infectious disease with a myriad of clinical features caused by the microorganism Treponema pallidum. Syphilis being termed as "the great imitator" can present a protean of clinical features. The complex interaction of HIV and syphilis is a subject of ongoing research and a co-infection with HIV may also alter the natural history of syphilis, sometimes leading to an overlap of primary and secondary features ${ }^{[3]}$ We present a case report of a simple looking yeast infection of the oral cavity that was found to be associated with HIV infection, which in turn was associated with syphilis. The case highlights the importance of the primary clinical presentation, which was oral leading to the diagnosis of HIV followed by a co-infection with syphilis. Many patients associated with oral manifestations of yeast infection have an underlying condition, but very few dentists and physicians order supplemental diagnostic tests associated, especially with immunocompromised states. The case also emphasizes the importance of prozone phenomenon in cases of secondary syphilis. Prozone phenomenon is seen in $<2 \%$ cases of secondary syphilis although in HIV-infected individuals, higher proportion can be affected, ${ }^{[4]}$ making it imperative to test for prozone phenomenon in the HIV syphilis co-infected population.

\section{CASE REPORT}

A 26-year-old unmarried male reported to the outdoor Department of Oral Medicine and Radiology with a 2-week history of burning sensation and redness of tongue with altered taste acuity. Oral examination of the patient revealed diffuse loss and atrophy of the filiform papillae on the dorsum of the tongue with insignificant changes of the oral mucosa [Figure 1]. Extraoral examination at this time was insignificant. A provisional diagnosis of erythematous candidiasis was made, and the patient was prescribed clotrimazole $1 \%$ weight/volume thrice a day for 1 -week.${ }^{[5]}$ Five days later, the patient returned without any resolution of the tongue lesions and neither any improvement of the burning sensation. Furthermore, he complained of systemic symptoms of lethargy, malaise, mild fever and difficulty in swallowing along with psoriasiform scaly lesions on the palms and soles and few violaceous plaques on the body. Detailed intraoral examination disclosed that the lesions of the tongue had extended into pharynx portraying a picture of oropharyngeal candidiasis. This raised suspicion and on further

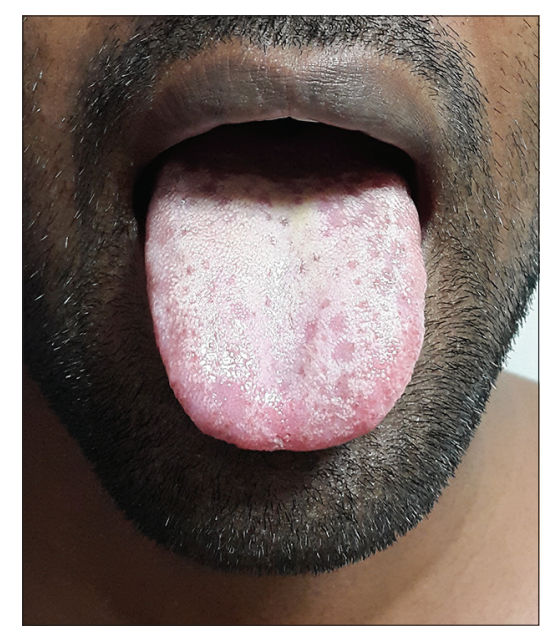

Figure 1: Diffuse loss and atrophy of the filiform papillae on the dorsum of tongue

investigating revealed a sexual history positive for multiple contacts, all unprotected and paid, last about 2 months back. The patient was heterosexual. On this second visit, enzyme linked immunosorbent assay for HIV 1 and 2 and Veneral Disease Research Laboratory (VDRL) were ordered. A couple of days later the patient came with the results, which showed positivity for HIV 1 and a negative VDRL test. This lead to a diagnostic and therapeutic dilemma and the patient was referred to the Department of Dermatology where the scales observed were silvery and densely adherent to the plaques [Figure 2]. Few violaceous plaques were seen on the thighs, chest $(2-3 \mathrm{~cm})$ and the scrotal area about $(1-2 \mathrm{~cm})$ in diameter [Figure 3]. The palmo-plantar lesions were tender on applying pressure with a blunt edge of a pin (positive Buschke-Ollendorf's sign). Patient also presented generalized lymphadenopathy. There was no evidence of condyloma lata and genital ulcer. There was no associated organomegaly. Hair, nails and other mucosal areas were normal. Patients past history was significant for a single genital ulcer 2 months back, which healed spontaneously. T. pallidum Particle Agglutination Assay (TPHA) was done and found to be positive. The VDRL test previously yielded nonreactivity but on informing the lab about the possibility of secondary syphilis by the dermatologist, prozone phenomenon (with a need to perform the test in higher dilutions), yielded a positive VDRL in 1:64 dilutions. Skin biopsy from a plaque on the chest showed a moderately dense superficial and deep perivascular and periappendageal infiltrate of lymphocytes, histiocytes, and plasma cells. The infiltrate in the upper dermis was present close to the epidermis in a patchy lichenoid pattern. The epidermis showed mild hyperplasia, spongiosis with neutrophils and focal parakeratosis [Figure 4]. Cerebrospinal fluid VDRL was non-reactive. Routine hemogram, liver and 


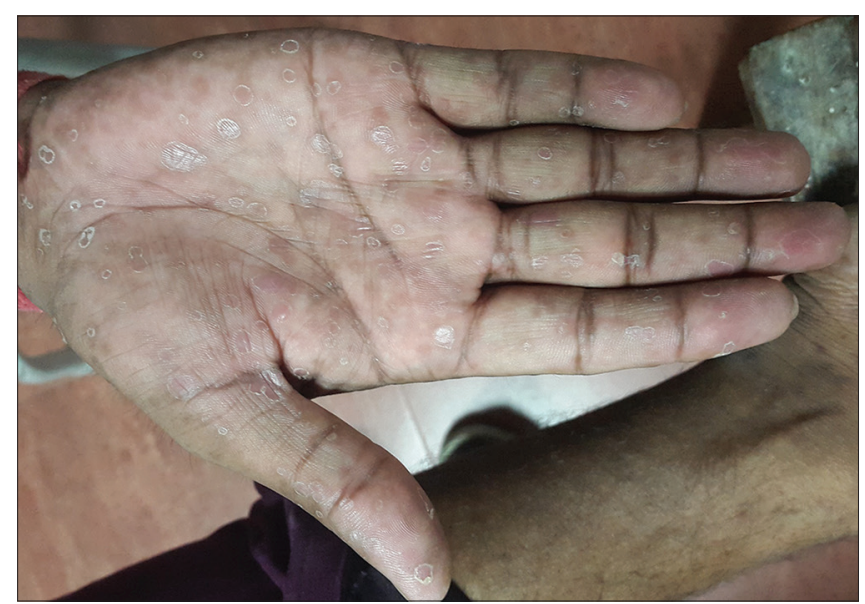

Figure 2: Psoriasiform scaly lesions of the palm

kidney function tests were all within normal limits. Peripheral blood CD4+ T cells counts of the patient were 737 cells $/ \mathrm{mm}^{3}$.

Thus, on the basis of personal history, clinical manifestations, positive VDRL test (prozone phenomenon) and biopsy from lesion site, a final diagnosis of HIV-1 infection co-infected with secondary syphilis and superimposed erythematous candidiasis of tongue was made.

\section{DISCUSSION}

Candidiasis is a fungal infection caused by yeast-like organism Candida albicans. Candida exists in two forms namely pseudo-hyphae, and yeast. ${ }^{[6]}$ The organism is a common dweller of the oral cavity, gastrointestinal tract and vagina of normal people. However, when the conditions are appropriate, the nonpathogenic yeast forms are transformed into pathogenic hyphae form. It lies as a commensal and waits for the host's defense systems to weaken. It can be classified into two major categories namely primary oral candidiasis-exclusively limited to oral tissues and secondary oral candidiasis - as a manifestation of systemic mucocutaneous candidiasis. It can present as acute and chronic forms with clinical patterns observed as pseudomembranous, erythematous, hyperplastic and angular cheilitis, and the first two variants being closely associated with immunosuppressive conditions like HIV ${ }^{[6]}$

Some $52 \%$ of HIV-infected individuals have Candida lesions in the early stages of infection. ${ }^{[7]}$ Since the detection of HIV and AIDS in 1981, it has been reported worldwide and the preceding couple of decades have seen a rise of HIV infections to pandemic proportions. The syndrome denotes a lethal viral infection producing some primary and overwhelming

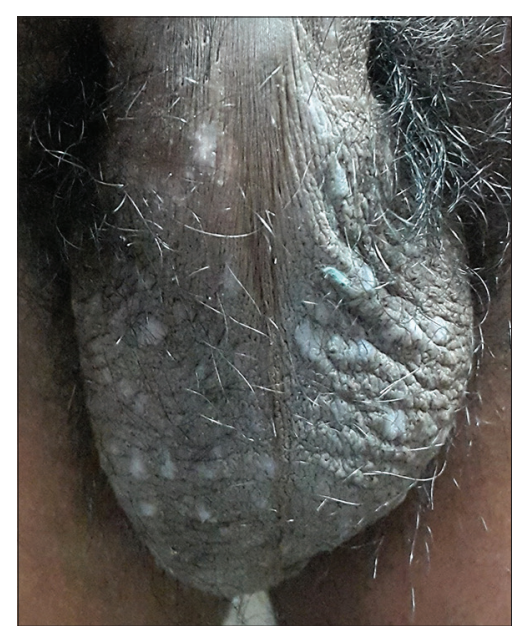

Figure 3: Violaceous plaques on the scrotal area (approximately $1-2 \mathrm{~cm}$ ) in diameter

secondary manifestations due to profound immunosuppression. HIV targets cells having CD4 surface antigen, which is primarily found on T-Helper cells causing progressive immunosuppression leading to opportunistic infections, neoplasms, and neurologic manifestations. In HIV-infected individuals, the virus can be found in many body fluids including serum, blood, saliva, semen, tears, urine, breast milk, ear, and vaginal secretions. The main routes of transmission are sexual contact, intravenous drug abuse, and perinatal exposure. With a relegated immune response, an HIV-infected individual is likely to contact sexually transmitted diseases more frequently, one such malady being syphilis. It is caused by T. pallidum. The offending organism is a Gram-positive, motile, microaerophilic spirochete. The organism is extremely vulnerable to drying hence the primary mode of transmission is sexual contact and from mother to child.

According to World Health Organization, more than 10.6 million cases of syphilis occur each year out of which 3.0 million cases occur in the South East Asia Region. ${ }^{[8]}$ Since both syphilis and HIV are transmitted sexually, it is no surprise that a significant number of patients are affected with both. ${ }^{[3]}$ HIV makes it more likely for syphilis to present with atypical features and furthermore overlapping of syphilitic stages. This makes it important for the dental and medical practitioners to be aware of how syphilis may appear in patients with underlying HIV infection. The pillar of diagnosis for syphilis detection in the non-HIV infected adult is serology. ${ }^{[9]}$ However, these tests may yield a false positive and false negative serology result in an individual infected with HIV. ${ }^{[10]}$ False-negative nontreponemal antibody tests are seen in approximately $2 \%$ of patients and are due to the prozone effect where obstructive antibodies or very 


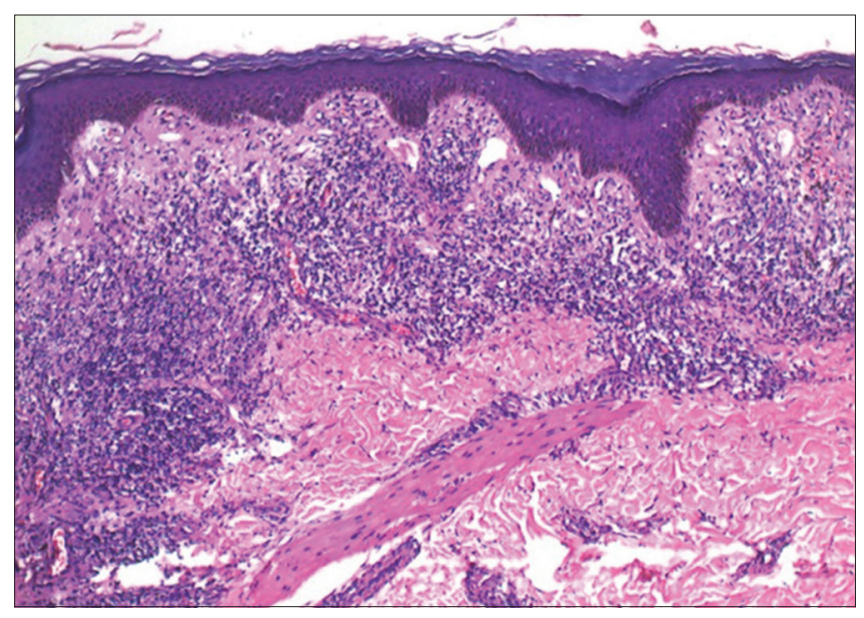

Figure 4: Biopsy of the epidermis showing mild hyperplasia, spongiosis with neutrophils and focal parakeratosis

high antibody titers interfere with the assay. ${ }^{[4]}$ This effect is observed when anomalous B-cell behavior causes hyper-responsiveness to antigenic stimulation, leading to excess antibody production.

When such conditions arise, a diagnostic as well as therapeutic catch 22 situation arises as was perceived in this case. The dilemma in our case was whether to treat the patient for the present cutaneous manifestation with Penicillin or to consider it as a case of TPHA persistent positivity due to the older ulcer of primary syphilis. We were also unsure whether lesions were of secondary syphilis or any nonspecific mucocutaneous manifestation of HIV. TPHA is a test, which once positive remains positive lifelong in spite of treated cases of syphilis whereas VDRL is the test that determines whether a patient needs to be treated, or not. The only usefulness of TPHA is to differentiate true positive from false positive in standard nontreponemal tests. We discussed with the lab about the possibility of secondary syphilis and to repeat the test in higher dilutions and voila, this time VDRL tested reactive in 1:64 dilutions. After reaching a final diagnosis of HIV-1 co-infection with secondary syphilis with superimposed erythematous candidiasis of tongue, the treatment plan was executed. Patient was treated with 3 weekly intramuscular injections of benzathine penicillin in a dose of 2.4 million units after sensitivity test. ${ }^{[3]} \mathrm{He}$ was also administered ketoconazole $200 \mathrm{mg}$ OD for 15 days for the management of oral lesions. Patient was counseled regarding safer sexual practice. Partner notification and contact tracing was done for the patient. He was also referred to the antiretroviral therapy center. The patient was followed weekly and then monthly with significant resolution of clinical lesions at the end of 2 weeks [Figure 5] and declined titers of VDRL to 1:4 at the end of 6 months. Our case

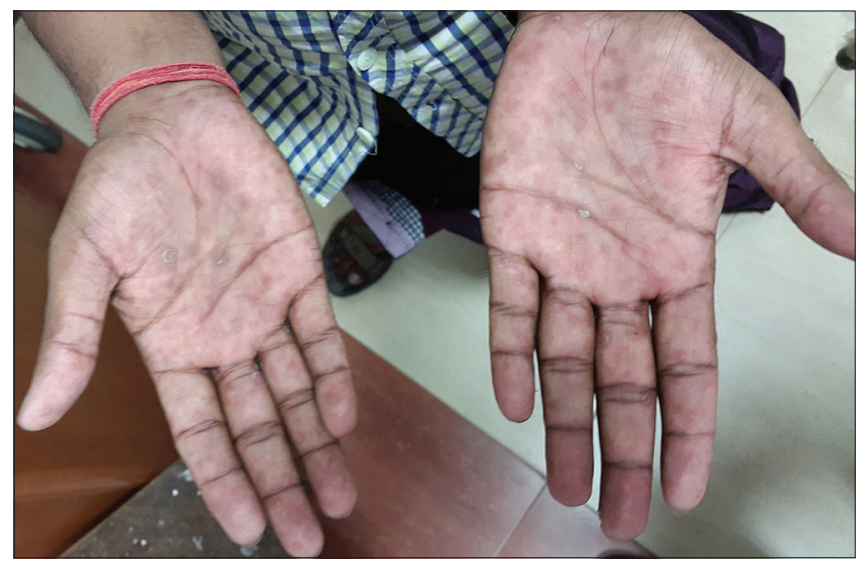

Figure 5: Significant resolution of clinical lesions at the end of 2 weeks

stresses the importance of diagnostic and therapeutic implications when patients present with such puzzling plethora of manifestations, which can baffle even the most trained eye and when the stakes are too high.

\section{Financial support and sponsorship} Nil.

\section{Conflicts of interest}

There are no conflicts of interest.

\section{REFERENCES}

1. Schubert MM. Oral manifestations of viral infections in immunocompromised patients. Curr Opin Dent 1991;1:384-97.

2. Dodd CL, Greenspan D, Katz MH, Westenhouse JL, Feigal DW, Greenspan JS. Oral candidiasis in HIV infection: Pseudomembranous and erythematous candidiasis show similar rates of progression to AIDS. AIDS 1991;5:1339-43.

3. Lynn WA, Lightman S. Syphilis and HIV: A dangerous combination. Lancet Infect Dis 2004;4:456-66.

4. Taniguchi S, Osato K, Hamada T. The prozone phenomenon in secondary syphilis. Acta Derm Venereol 1995;75:153-4.

5. Ellepola AN, Samaranayake LP. Oral candidal infections and antimycotics. Crit Rev Oral Biol Med 2000;11:172-98.

6. Samaranayake LP. Oral mycoses in HIV infection. Oral Surg Oral Med Oral Pathol 1992;73:171-80.

7. Felix DH, Wray D. The prevalence of oral candidiasis in HIV-infected individuals and dental attenders in Edinburgh. J Oral Pathol Med 1993;22:418-20

8. Rowley J, Toskin I, Ndowa F. Global incidence and prevalence of selected curable sexually transmitted infections, 2008 (WHO, Geneva): World Health Organization; 2012.

9. Larsen SA, Steiner BM, Rudolph AH. Laboratory diagnosis and interpretation of tests for syphilis. Clin Microbiol Rev 1995;8:1-21.

10. Augenbraun M, Rolfs R, Johnson R, Joesoef R, Pope V. Treponemal specific tests for the serodiagnosis of syphilis. Syphilis and HIV Study Group. Sex Transm Dis 1998;25:549-52.

\begin{tabular}{|l|l|}
\hline \multicolumn{3}{|c|}{ Access this article online } \\
\hline Quick Response Code: & \\
\hline
\end{tabular}

